\title{
Artikel 22a KVG - der Hund der Baskervilles
}

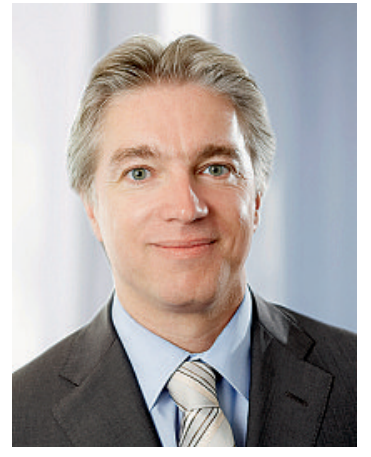

Still und fast heimlich ist er gekommen und für Unruhe und Lärm wird er sorgen: der Artikel 22a des KVG [1]. Eingeführt am 1. Januar 2009, überfordert er momentan alle: die Ärzteschaft, weil die Kriterien der geforderten Information unklar sind, den Bund, weil die Erhebung der benötigten Daten alles andere als banal ist, und die Versicherer, weil sie sich interessante Zusatzinformationen erhoffen, aber nicht wissen, was sie wissen möchten.

Es ist etwa so wie beim Hund der Baskervilles: Alle haben Schreckliches über ihn gehört. Aber niemand hat ihn gesehen und niemand kann seine Gefährlichkeit wirklich abschätzen.

Nimmt man die von Artikel 22a KVG geforderten Daten im Stile Sherlock Holmes' genauer unter die Lupe, sind gewisse Informationen einfach erhältlich und genauso problemlos publizierbar. Wenn es dann Richtung Umfang und Kosten von erbrachten Leistungen geht und der Ertrag sowie das finanzielle Betriebsergebnis einer ärztlichen Praxis öffentlich gemacht wird, muss man sich schon fragen, was der gesundheitspolitische Nutzen von solchen Datenpublikationen ist. Gänzlich unklar ist der Artikel, wenn es um medizinische Qualitätsindikatoren geht, die in keiner Art und Weise näher definiert sind.

\section{Artikel 22a KVG überfordert sowohl die Ärzteschaft als auch den Bund und die Versicherer}

In dieser Unklarheit liegt allerdings auch die Chance für die FMH. Die Verbindung der Schweizer Ärztinnen und Ärzte bespricht gegenwärtig die zentralen Fragen zur Umsetzung dieses KVG-Artikels mit den betroffenen Organisationen und erarbeitet Lösungsmodelle. Ziel dieser Diskussionen ist es, mit einem vernünftigen Aufwand - unter Berücksichtigung bereits vorhandener Daten - dieser gesetzlichen Datenlieferungspflicht nachzukommen. Als Segen erweisen sich hier, einmal mehr, die ärzteeigenen Daten aus den Trustcentern und der ROKO, die unter anderem über NewIndex aufbereitet werden und mit denen ein Teil der geforderten
Daten abgedeckt werden kann. Die Knacknuss wird aber, wen wundert's, die Definition der zu liefernden Qualitätsindikatoren sein. Die FMH versucht auf verschiedenen Wegen, zusammen mit den anderen Akteuren im Gesundheitswesen, eine sinnvolle und nutzbringende Lösung zu erarbeiten. Einerseits sind wir daran, in einer Arbeitsgruppe mit Vertretern von santésuisse und von Versicherungen mögliche

\section{Welche Qualitätsindikatoren sind nutzbringend, welche nicht?}

Indikatoren einzugrenzen. Andererseits ist die Umsetzung des Art. 22a KVG auch Thema in den Arbeitsgruppen mit dem BAG zur Konkretisierung der Qualitätsstrategie des Bundes. Eines ist sicher: Eine einfache, gute Lösung zu finden, ist enorm schwierig, und ein schrittweise konzertiertes Vorgehen ratsam. Anzunehmen ist, dass die ersten veröffentlichten Zahlen, zu erwarten im Jahr 2013, wohl noch nicht den ganzen Bereich von Art. 22a KVG abdecken, und eine sukzessive Erweiterung der Datenpublikation in Richtung der komplexeren Indikatoren und Kennzahlen stattfinden wird.

Wer weiss, was bis dann noch alles in der Gesundheitspolitik geschieht ... schliesslich wurde der Hund der Baskervilles am Ende auch erschossen.

Dr. med. Daniel Herren MHA, Mitglied des Zentralvorstandes der FMH, Verantwortlicher für das Ressort DDQ

1 Artikel 22a KVG: «Die Leistungserbringer sind verpflichtet, den zuständigen Bundesbehörden die Daten bekannt zu geben, die benötigt werden, um die Anwendung der Bestimmungen dieses Gesetzes über die Wirtschaftlichkeit und Qualität der Leistungen zu überwachen. Namentlich sind folgende Angaben zu machen:

a. Art der Tätigkeit, Einrichtung und Ausstattung, Rechtsform;

b. Anzahl und Struktur der Beschäftigten und der Ausbildungsplätze;

c. Anzahl und Struktur der Patientinnen und Patienten in anonymisierter Form;

d. Art, Umfang und Kosten der erbrachten Leistungen;

e. Aufwand, Ertrag und finanzielles Betriebsergebnis;

f. medizinische Qualitätsindikatoren.» 